\title{
A Kind of PM2.5 Purification Detection Device Based on Solar Thermoelectric Power Generation
}

\author{
Wang Huasheng, Xu Qiaobian, Wang Mu \\ North China Electric Power University (Baoding), Baoding. 071000, China
}

\begin{abstract}
Keywords: temperature difference power generation; PM2.5 purification; PM2.5 detection; wind speed optimization.
\end{abstract}

\begin{abstract}
In order to improve the serious haze weather, this paper puts forward a kind of PM2.5 purification detection device based on solar thermoelectric power generation. Combined with the actual operating conditions, the thermodynamic model of the temperature difference power generation device is established by theoretical derivation; the air speed optimization scheme is obtained through numerical calculation of temperature difference generator; the purification performance can be determined through experiment. This paper also analyzes thedevice in practicability and economy which proves that the device can operate stably and has a certain application prospect.
\end{abstract}

\section{Introduction}

China's air pollution is very serious. Among the 161 cities monitored, there are more than 46 cities occurring severe pollution. Besides,16 cities are seriously polluted. With the deterioration of air quality,haze weather increases. Haze weather not only affects the atmospheric environmental quality but also endangers human health. Aiming at the shortcomings of the more common air purifiers on the market, we designed a set of combination of solar thermoelectric power generation and hand mechanical power of the new air PM2.5 detection and purification device: making use of clean energy--solar energy and mechanical energy to generate electricity for the use of equipment, which is beneficial to environment; combining detection and purification system, which makes the device fit flexibly and break up easily. The device can be used in the detection of indoor and outdoor protection and air purification. What's more, the detection part can be removed to carry flexibly and conveniently. Based on the theoretical derivation, experimental determination, graphic simulation and numerical calculation, this paper establishes the thermodynamic model of the temperature difference power generation device and determines the electric power of the temperature difference generator.It concludes the optimization scheme of the temperature difference generator.This paper not only analysis the effect of air purification but also proves the device can run normally.

\section{The Basic Principles}

This device mainly include three parts: solar thermoelectric generator module, PM2.5 detection module and PM2.5 air purification. The principle of each module is as follows:

Solar Thermoelectric Power Generation Module.

Beam goes through the Fresnel lens and focuses on the aluminum sheet to collect heat . Heat conducts through the aluminum and graphite flake to the hot junction of the solar thermoelectric power generation chip, and then through the solar thermoelectric generator chip and the graphite piece to the hotjunction. Fan makes cold junction and the air form forced convection heat transfer, to reduce the temperature of the cold junction of the solar thermoelectric power generation chip, forming temperature difference between the hot and the cold junctions of the solar thermoelectric power generation chip. Solar thermoelectric power generation chip uses the Seebeck effect to output power. After being rectified, filtered and stabilized, the power is stored in the battery. Then, the battery can supply power to the purify part, as well as the test system. This module transfers the sun's heat directly into electricity and its specific structure diagram is shown in Figure 1. 


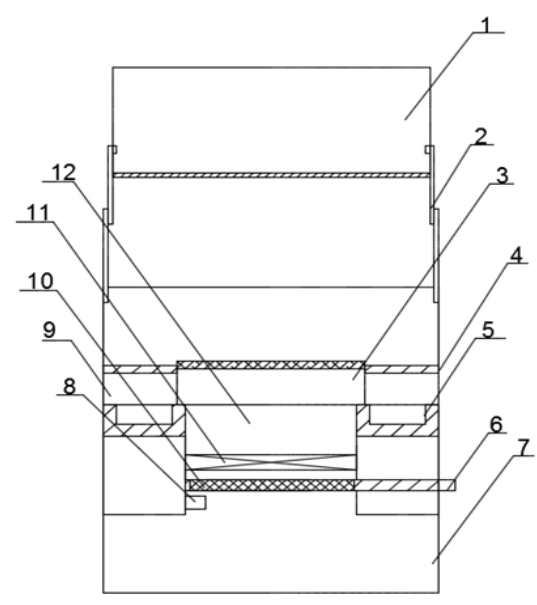

Figure 1.The specific structure diagram of the device

1- Fresnel lens;2-Bracket;3—Solar thermoelectric power generation device;4-The sealing cover;5-Tank;6—Filter tray ;7-Air outlet;8—Negative-ion generator;9—Air inlet;10—HEPA filter;11-Fan;12-Air duct;

\section{Air Purification Module.}

Air cooling system and PM2.5 purification link share a fan. The outside air skims over the tank surface, under the action of fan to pump the air. After being humidified, the air flows into the device, through the HEPA filter and negative-ion generator, effectively reducing the content of PM2.5 in the air. Finally, the air is discharged into the surrounding environment and the device accomplishes the purification task.

\section{PM2.5 Detection Module.}

Detection module consists of hand crank part and PM2.5 detection part. In hand-operated mechanical power part, turning the cylindrical handle to drive the gear transmission mechanism, then further drive the motor spindle rotation to produce electricity for the whole PM2.5 test circuit. Dust sensors detectthe light signal reflected by the dust in the air, convert it into analog voltage signal and pass it to the single chip microcomputer. Based on the analog voltage signal, SCM approximately calculates the value of PM2.5 and displays the value on the display circuit, accomplishing the PM2.5 detection task.

\section{Performances}

\section{Analysis of The Performance of Thermoelectric Power Generation.} Performance parameters of thermoelectric power generation.

The model of thermoelectric power generation chip is TEG1-199-1.4-0.5, and the total number of thermoelectric power generation chip is 9 which are arranged between two pieces of graphite sheet in the way of $3 \times 3$. In order to get the curve of output power with the change of temperature difference between the cold junction and hot junction ,we close the purify modular, that is to say , just make the device work in the way of storing electric energy. According to the data we get by measuring the different temperature difference between the cold junction and hot junction of thermoelectric power generation chip and the output power under those different conditions, we get the curve of the output power, as Figure 2 shows: 


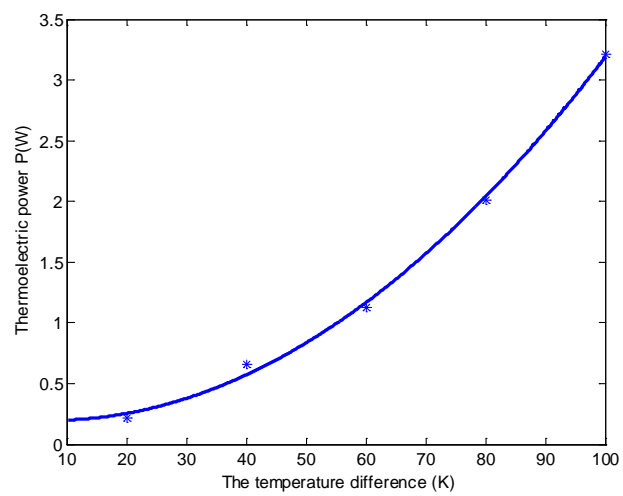

Figure 2. The thermoelectric power with variation of temperature difference

According to the Figure 2, we can draw the conclusion that with the increase oftemperature difference between hot and cold junction of thethermoelectric power generation chip, power generation also increase. At the same time, the amount of increase becomes larger. So to increase the output power of the thermoelectric power generation chip, we need to increase the temperature difference between the cold junction and hot junction, which is the key factor to design the device and also the guarantee that the device can work well.

\section{Thermodynamic Model.}

To simplify the problem,we make the following assumptions in the process of modeling:

(1) There is no difference of the 9 thermoelectric power generation chips, that is to say the thermal conductivity, length, cross-sectional area and any other parameters are all same.

(2) Ignore the thermal radiation of hot and cold junction and the system's heat exchange with the surroundings ;

(3) The heat conducted by the graphite sheet is uniform in different area of the graphite sheet.

And the schematic diagram of the temperature difference power generation device just as the Figure 3 shows.

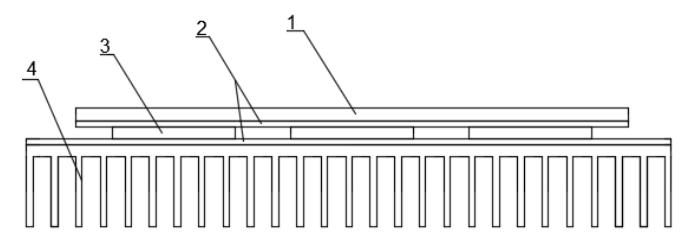

Figure 3 .The schematic diagram of the temperaturedifference power generation device 1-Aluminum sheet;2-Graphite sheet;3-Thermoelectric power generation sheet;4-Air-cooling fin.

In the practical research, we just analysis the steady heat conduction of the device. The beam through the final lens focuson the heat collecting aluminum sheet, and the heat transfer rate of the aluminum sheet is $\phi$ which is also the total heat that the device absorbs. One part of the heat flows through the thermoelectric power generation chip,then converses into electrical power, of which the power is $P$ and the conversion efficiency is $\eta_{1}$. The other flows from the cold junction, the air-cooling fin, and lost to the surroundings. We use the letter $\phi_{1}$ to describe the heat transfer rate that is used by thermoelectric power generation chip and $\phi_{2}$ to describe the lost part.

So, the total heat transfer rate conducted by aluminum sheet is:

$$
\phi=\frac{T_{1}-T_{2}}{R_{1}}=\frac{T_{1}-T_{2}}{\frac{\delta_{1}}{A_{1} \lambda_{1}}}
$$

Where $A_{1}$ is the area of the aluminum sheet; $T_{1}$ is the temperature of the aluminum sheet's upper surface $; T_{2}$ is the temperature of the aluminum sheet'slower surface ; $R_{1}$ is thermal resistance of the aluminum sheet; $\delta_{1}$ is the thickness of aluminum sheet $; \lambda_{1}$ is the thermal conductivity of the 
aluminum sheet.

The temperature difference between the hot and cold junction of thermoelectric power generation chip:

$$
\Delta T=T_{3}-T_{4}
$$

Where $T_{3}$ is the temperature of thermoelectric power generation chip's hot junction; $T_{4}$ is the temperature of thermoelectric power generation chip's cold junction.

We can get the relation between the temperature difference and the rate of power generation is:

$$
P=f(\Delta T)
$$

The heat that the thermoelectric power generation sheet consumes to generation electricity is:

$$
\phi_{1}=\frac{P}{\eta_{1}}
$$

Look up the thermal physical properties of the dry air at standard atmospheric pressure, and get the Nussle number by the feature correlation equation:

$$
\mathrm{Nu}=\frac{0.4637 \mathrm{Re}^{\frac{1}{2}} \mathrm{Pr}^{\frac{1}{3}}}{\left[1+(0.0207 / \mathrm{Pr})^{\frac{2}{3}}\right]^{\frac{1}{4}}}
$$

So the convection heat transfer coefficient is:

$$
h_{1}=\frac{\lambda_{2}}{l_{2}} \mathrm{Nu}
$$

Where $\lambda_{2}$ is the thermal conductivity of air; $l_{2}$ is the length of the fin which is along the direction of the air-flow.

The heat flows lost to the surroundings from the air-cooling fin by convection is:

$$
\phi_{2}=\eta_{\mathrm{f}} \mathrm{h}_{1} \mathrm{~A}_{3}\left(\mathrm{~T}_{4}-\mathrm{t}_{0}\right)
$$

Where $\eta_{f}$ is efficiency of the fin; $h_{1}$ is the convective heat transfer coefficient of the fin; $A_{3}$ is the total area of the air-cooling fin that exchanges heat with cold air; $T_{4}$ is the temperature of thermoelectric power generation chip's cold junction; $t_{0}$ is the temperature of the cold air.

To simplify the calculation, we just take the area of the fin into account and ignore the area of the root of the fin. To a certain degree ,it is reasonable.

We can get the equation under the law of conservation ofenergy:

$$
\phi=\phi_{1}+\phi_{2}
$$

Through programming, numerical computing, we get the relationship between the air speed $v$ and the rate of power generation $p$ by the thermoelectric power generation chip, as the curve shows in Figure 4:

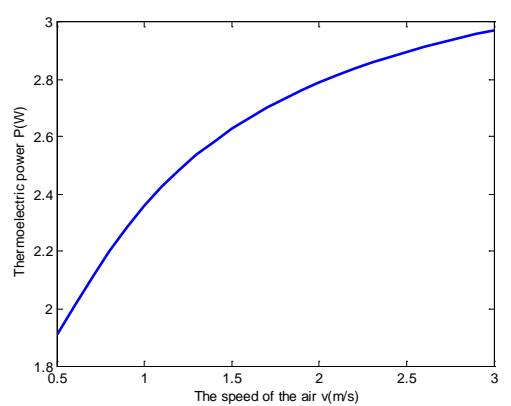

Figure 4. The relationship between the rate of power generation $p$ and the air speed $v$

According to the Figure 4, we can get the conclusion that with the increasing of the air speed, the rate of power generation of the thermoelectric power generation chip increases.For the reasons of that increasing the air speed is to increase the airdisturbance, which speeds up the convection heat transfer, and leads to the temperature decrease of the cold junction of the thermoelectric power generation chip.So the temperature difference between the hot and cold junction increases. AccordingFigure 2, the rate of power generation increases. 


\section{Device Optimization.}

In this unit, fan strengthens internal air disturbanceto speed up the air-cooling finto exchange heat with the surroundings which reduces the cold junction's temperature. The colder the cold junctionis, the greater the power outputs. The greater the air speed is, the greater the power outputs. At the same time, the greater the air speed is, the larger the energy consumption of the fan is. In order to get the maximum net power capacity, there is an optimal scheme in fan air speed.

On the basis of Figure 4, this paper comes up with the relation between the fan speed and net energy storage power considering the energy dissipation of the fan and other parts of the device, which is shown in Figure 5:

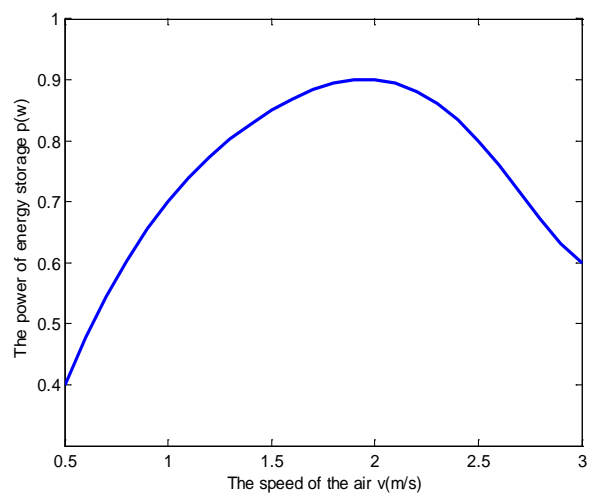

Figure 5 Net energy storage power changes with air speed curve

As the above curve shows, there is a optimal value in air speed of the thermoelectric power generation module, making the biggest net energy storage powerto ensure the high-efficiency and stability of the device. For the purposes of this device, the optimal air speed is between $1.8 \mathrm{~m} / \mathrm{s}$ and $2 \mathrm{~m} / \mathrm{s}$.

Performance Analysis of Air Purification.

The experiment was carried out under the conditionsbelow to analyzethe performance of purification module.

Environment temperature: $20 \pm 1.5{ }^{\circ} \mathrm{C}$;

The volume of the space tested: $11 \mathrm{~m}^{3}$;

The number of air outlet of the device: 2;

The size of air outlet: $182 \times 80 \mathrm{~mm}$.

At the beginning of the experiment, the concentration of PM2.5 for the airtight environment is $266 \mu \mathrm{g} / \mathrm{m}^{3}$.Start the device and change the fan speed to realize the change of air speed. The real-time monitoring PM2.5 concentration curve is shown in Figure 6.:

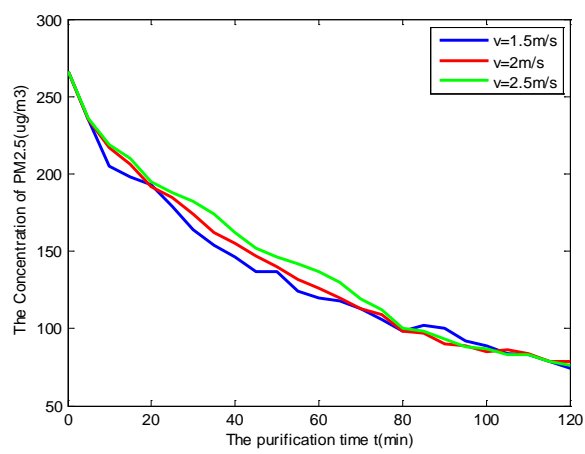

Figure 6 PM2.5 values under different wind speed variation over time

As is shown in the Figure 6,the thermoelectric power generation device can ensure purification module operate stably. There isno much difference in purification efficiency when the air speed changes among $1.5 \mathrm{~m} / \mathrm{s}, 2 \mathrm{~m} / \mathrm{s}, 2.5 \mathrm{~m} / \mathrm{s}$. What's more, there is no significant difference in the results and time of the purification. Namely in the range of the air speed allowed, the purification efficiency of this device is the same approximately. 


\section{Economic Benefits}

Cost.

The PM2.5 purification detection device based on thermoelectric power generation costs 400 450 Yuan averagely. Compared with the same purification ability products which cost thousandsYuanon the market, our deviceis much cheaper and has certain advantages.

Power Benefits.

Due to the uneven solar energy distribution, the solar radiation of different regions anddifferent moments is different. We conduct experiments to analyze the one-day capacity change of the equipment somewhere in NorthChina, the results are shown in Figure 7.

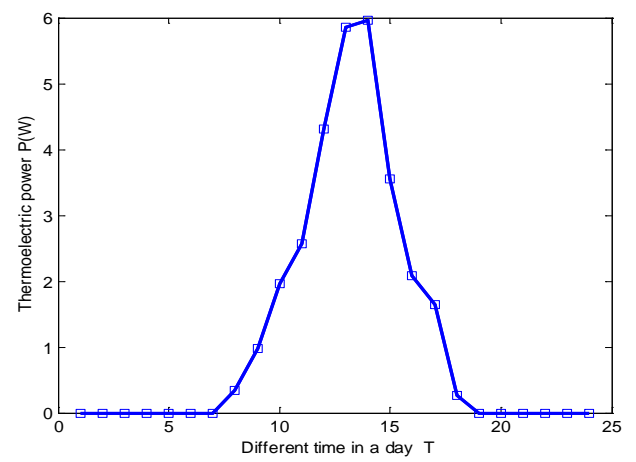

Figure 7. Output power generation over time

When the power $P$ is less than $0.8 \mathrm{~W}$,temperature difference power generation system is unable to supply power. There are two effective power point of time $t_{1}, t_{2}$. Time for effective power outputis $\left(t_{1}, t_{2}\right)$, total energy production is $W=\int_{t_{1}}^{t_{2}} p d T$.

By calculation, the averagedaily production of solar thermoelectric power.Thepower can make the purification work continuously and stably for 6.44 hours. Therefore the use of solar thermal power can not only ensure the stable operation of the device, but also help save energy and environmental protect the environment.

At the same time, there are mainly three kinds of patterns of the purification section: when the air does not need purification, it is the storage mode. We can take out of the filter structure, thus reducing the air flow resistance, strengthening the cold junctionheat dissipation and improving the efficiency of power generation. When the air needs purification, it is the storage and purification mode.Thermoelectric power generation deviceprovides electricity and the rest is stored in the batteries. When the air requires purification but thelight can not meet the power generation, the only energy is the energy stored in batteries. It is the purification mode. Compared the energy-saving type air detection and purification device with traditional instruments, the use of clean energy is environmentally friendly. Besides, the energy-saving effect is remarkable, making the device has greater market space and promotion value.

The purifying efficiency.

There is a space which is $11 \mathrm{~m}^{3}$ in volume and itsinitial even concentration of PM2.5 is $266 \mu \mathrm{g} / \mathrm{m}^{3}$.It takes 100 minutes for its concentration of PM2.5 to decrease $75 \%$, when the optimal fan speed is $2 \mathrm{~m} / \mathrm{s}$. As a result, the ventilation frequency is estimated to be 0.6 . Since it can meet the basic requirements when the ventilation frequency is 0.5 , this device has better purification efficiency in small space.

\section{The Improvement Schemes}

\section{Thermoelectric power generation optimization.}

Since the device can not guarantee that the aluminum sheet is heated evenly, 9 thermoelectric power generation chips are unevenly heated,which affects the efficiency of power generation.It can be optimized by controlling the thermoelectric power generation chip and aluminum sheet area ratio. 


\section{Optimization of heat transfer process.}

Because convection heat transfer with air and radiation heat transfer with the surrounding surface occur on the surface of the aluminum sheet .So we can put a transparent cover on the aluminum sheet or heighten the box body height to reduce the heat loss.

Noise optimization.

The device's running noise mainly comes from the fan and the friction between air flow and air duct. In order to reduce the noise, the inlet and outlet of the tuyere, the fan edge and the air duct can be chamfered, reducing the local resistance, thereby reducing the noise when running.

\section{Conclusions}

(1) Using solar thermoelectric generator to supply power is feasible and can ensure purification module to operate stably.

(2) Analysis, which is based on the experimental determination of purification performance, proves that this device has a certain effect of purification and it is practical and economical.

(3) The thermodynamic model of this device is established by theoretical derivation and we get the optimized air speed $1.8 \mathrm{~m} / \mathrm{s} \sim 2 \mathrm{~m} / \mathrm{s}$ by numerical calculation.

(4)Three kinds of purification modes not only ensure the PM2.5 purification, but also ensure the efficient production and storage of electrical energy, flexibly and conveniently.

\section{References}

[1]Reng Depeng,Jia Yang,Numerical simulation for operating characteristics of thermogenerator, Spacecraft Engineering,2008, 17(4):56-61(in Chinese).

[2]Jia Lei,Chen Zeshao, Research on thermal conductivity of thermoelectric generator, Acta Energies Solaris Sinica,2004,25(6):816-819

[3]Yang Yongjie,Zhang Yusheng, Design of a PM 2.5 detecting sensor, Transducer and microsystem,2014, 33(3).

[4]Deng Changsheng, the Principle and Application of Solar Energy, Chemical Industry Press,2010, 35(in Chinese)

[5]Xuan X C, Li D. Optimization of a combined thermionic-thermoelectric generator, Journal of Power Sources,2003, 115:167-170 